\title{
Dienogest-The Millennium Molecule!!
}

\author{
Shilpa Venkatesh* \\ Department of OBG, St. John's Medical College, India
}

*Corresponding author: Shilpa Venkatesh, Department of OBG, St. John's Medical

College, India, Tel: +919845726200; Email: shilpavenkatesh1280@gmail.com

Mini Review

Volume 2 Issue 2

Received Date: May 12, 2017

Published Date: July 12, 2017

\section{Introduction}

Dienogest (DNG) is a fourth generation progestin, being extensively used in a wide variety of clinical settings in gynecology. In order to write this comprehensive review, a literature search was performed to identify systematic reviews and randomised trials involving DNG. Databases searched included MEDLINE, PUBMED, Embase and the Cochrane library. The term 'dienogest' was associated with the following search terms: 'endometriosis', 'contraception', 'hormone replacement therapy', 'safety', 'efficacy'

\section{Pharmacology}

Dienogest (DNG) is a 19-nortestosterone derivative (a C-19 progestogen) with a cyanomethyl instead of an ethinyl group at the C-17 position. Properties derived from its C-19 derivative structure include its short plasma half life, of about $10 \mathrm{~h}$ (which means the drug is not accumulated), and its high oral bioavailability, of more than $90 \%$. DNG also has some properties typical of other progesterone derivatives, including a lack of effect on the metabolic and cardiovascular systems, and considerable antiandrogenic activity [1,2]. DNG has no antiestrogenic activity, which is explained in its role in the treatment of endometriosis [3]. It is exclusively protein bound (albumin-90\%, free-10\%). The lack of DNG binding to sex hormone binding globulin means it does not displace testosterone, resulting in the androgenic effects observed with other progestins. It is metabolized by hydroxylation and conjugation, predominantly in the liver [4]. DNG is available in the dose of $2 \mathrm{mg}$ /day (Figure 1, Table 1).

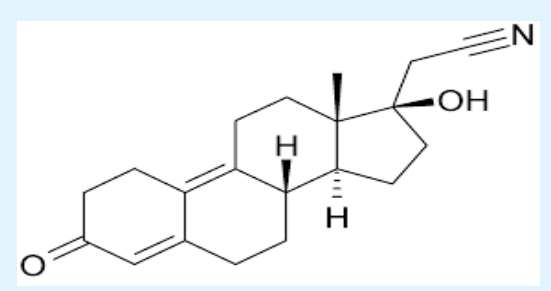

Figure 1: Structure of Dienogest.

\begin{tabular}{|c|c|}
\hline $\begin{array}{c}\text { Typical of other } \\
\text { progesterone derivatives }\end{array}$ & $\begin{array}{c}\text { Attributed to its C19 } \\
\text { Derivative Structure }\end{array}$ \\
\hline $\begin{array}{c}\text { Lack of CVS and metabolic } \\
\text { effects }\end{array}$ & Short plasma half life \\
\hline Anti-androgenic activity & $\begin{array}{c}\text { High endometrial efficacy } \\
\text { High oral bioavailability }\end{array}$ \\
\hline
\end{tabular}

Table 1: Properties of Dienogest.

\section{Adverse Effects}

In a safety cohort of 727 women [5], the most frequently reported adverse effect with DNG was headache (9\%), acne (5.1\%), nausea (4.2\%), and weight gain (3.6\%). Changes in menstrual bleeding patterns were also common in these trials. After 9-12 months of use, bleeding was regularized in $22 \%$, but $28 \%$ reported amenorrhoea, oligomenorrhoea in $24 \%$ and polymenorrhoea in $2.7 \%$.

Clinical settings in which dienogest is currently used

- Endometriosis

- In contraception

- Hormone replacement therapy (HRT)

\section{Dienogest in Endometriosis}

Mechanism of action: DNG reduces endometriotic lesions through a number of biological mechanisms. It is associated with relatively moderate inhibition of gonadotrophin secretion, leading to a modest reduction in the endogenous production of estradiol [5]. When given continuously, dienogest induces a hypoestrogenic, hypergestagenic local endocrine environment, causing a decidualization of endometrial tissue followed by atrophy of the endometriotic lesions [6]. In exploratory models of 
endometriosis, dienogest also demonstrates antiproliferative, anti-inflammatory, and anti-angiogenic effects $[7,8]$.

\section{Dienogest versus GnRh Agonists}

Pain relief: A large $\mathrm{RCT}(\mathrm{N}=252)$ was carried out, in which 124 women were randomised to receive DNG and 128 women received leuprolide acetate( LA) [9]. The VAS (Visual analog scale) score was selected as the primary efficacy variable in this study because the VAS is an appropriate and well established tool for the measurement of pelvic pain associated with endometriosis [10]. Absolute reductions in VAS score from baseline to week 24 were $47.5 \mathrm{~mm}$ with DNG and $46.0 \mathrm{~mm}$ with LA. This finding was of high clinical relevance, as pelvic pain is one of the most important symptoms of endometriosis and because agents in the $\mathrm{GnRh}$ agonist class are widely considered a reference standard treatment for improving these symptoms [11].

Hypoestrogenic effects: It is a known fact that GnRH agonists produce hypogonadotrophic hypogonadism. An interesting hypothesis was proposed by Barbieri [12], way back in 1992. It stated that, 'a concentration of estradiol that will partially prevent bone loss may not stimulate endometrial growth'. DNG maintains estrogen levels within the therapeutic window for endometriosis that is $30-50 \mathrm{pg} / \mathrm{ml}$. Hence, the incidence of hypoestrogenic effects is less with DNG when compared to LA [9].

Bone mineral density (BMD) reduction: $\mathrm{GnRH}$ agonists cause significant loss in trabecular and cortical bone, particularly lumbar spine and femoral neck. This may even exceed $1 \%$ per month [13]. On the other hand, DNG showed no change in the mean lumbar BMD during the treatment period [9], demonstrating minimal changes in bone turnover/bone resorption markers.

\section{Dienogest versus Other Progestins (Norethindrone Acetate)}

Dienogest as the first-choice progestin for the medical therapy of symptomatic endometriosis does not confer additional benefits compared with norethindrone acetate in terms of pain relief, health-related quality of life, or sexual functioning [14]. Effectiveness of either of the progestins is greatly affected by economic aspects.

\section{Fertility Considerations with DNG Use}

Based on the current evidence, dienogest causes complete inhibition of ovulation at a daily dose of $2 \mathrm{mg}$
$[15,16]$. However, dienogest monotherapy was not developed as a contraceptive, and women taking dienogest as a treatment for endometriosis are advised to use non-hormonal methods of contraception [17]. The ovarian activity resumes rapidly (range 1-43 days) after cessation of dienogest [15]. Successful pregnancy has been reported in women with endometriosis following the cessation of dienogest treatment $2 \mathrm{mg}$ daily for duration of up to 1 year $[18,19]$.

Long Term Safety Profile of DNG in Endometriosis: Two large trials have been performed in Europe and Japan, to investigate the role of DNG in the long term treatment of endometriosis. The European trial offered DNG for an overall treatment period of 65 weeks [19]. The intensity of pain showed significant, sustained improvement during this long term study. In addition, during the 24 week treatment free period following the long term study, visual analog scores increased only moderately, suggesting that DNG induces a beneficial effect that may persist even after treatment cessation.

The results of the European study were supported by a 52 week, non-randomized trial of DNG conducted in Japan on 135 women with confirmed endometriosis [20]. Patient satisfaction with DNG at the end of treatment was high, with $88.9 \%$ of women responding that they were "certainly willing" or "would prefer" to use DNG again.

\section{Dienogest as a Contraceptive}

Estradiol valerate/dienogest (E2V/DNG) is a 4-phasic oral contraceptive approved for the prevention of pregnancy. The 4-phasic design allows for acceptable cycle control and in efficacy trials of estradiol valerate/dienogest in women aged 18-35 years, the Pearl Index ranged from 0.40 to 1.64 , a range comparable to that of other combination oral contraceptives [21].

In most combination oral contraceptives, the estrogen component is responsible for providing cycle control and stabilizing the endometrium for an acceptable bleeding pattern. E2V, unlike ethinyl estradiol, did not provide an adequate level of endometrial stability, as evidenced by breakthrough bleeding rates in preliminary studies [22]. Hence, E2V containing combination oral contraceptives did not reach the market until this novel combination of E2V and DNG was evaluated.

This combination integrates an estrogen step-down and a progestin step-up approach in a quadri-phasic regimen (Figure 2). 
- 26 active tablets - estrogen step-down, progestogen step-up

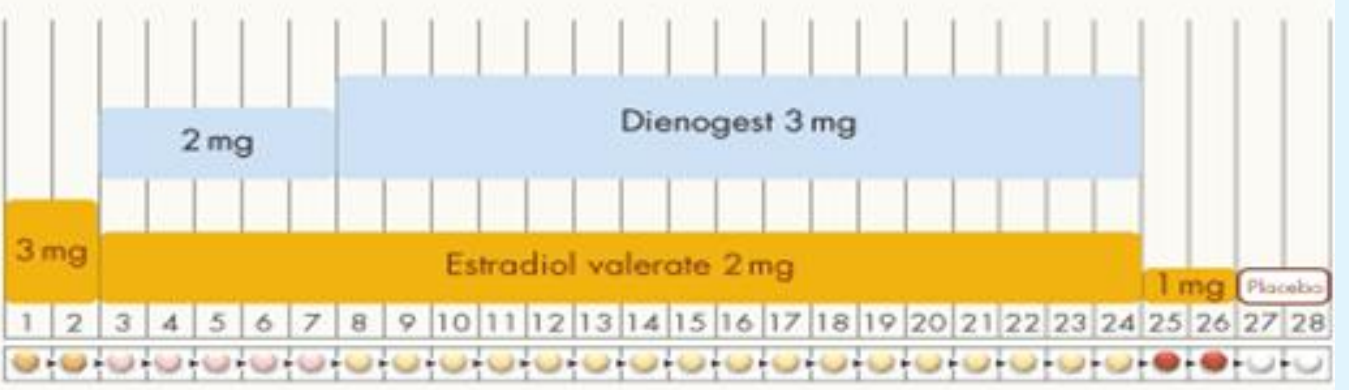

Figure 2: Daily doses of E2V and DNG in the quadriphasic regimen.

The American College of Obstetrics and Gynecology recommends that patients with anovulatory or ovulatory bleeding be treated with a combination oral contraceptive if they require contraception [23,24]. E2V/DNG is an appropriate first choice in heavy menstrual bleeding, for most premenopausal women who have a need for contraception, do not have contraindications to estrogen therapy, and do not desire LNG-IUD insertion. Use of E2V/DNG for six months has led to significant reduction in heavy menstrual bleeding with an average $65 \%$ reduction in mean blood loss [23].

\section{Dienogest in HRT}

A combination of $2 \mathrm{mg}$ estradiol valerate with $2 \mathrm{mg}$ dienogest (E2V/DNG) is the first continuous combined postmenopausal hormone replacement therapy (HRT) preparation to contain a progestogen with substantial anti-androgenic activity. A study of its clinical efficacy and safety in a comparative study versus a combination of 2 mg estradiol with $1 \mathrm{mg}$ norethisterone acetate (E2/NETA) has shown both preparations to be highly effective in achieving a rapid response in women with postmenopausal symptoms [25]. E2V/DNG is a novel HRT preparation that has a highly favorable bleeding profile.

\section{Conclusion}

Dienogest is a novel drug in the treatment of endometriosis, with an efficacy comparable to GnRh agonists. It is orally administered (unlike GnRh agonists) and more reasonably priced.Its role in contraception and HRT seems promising.

\section{References}

1. Herkert O, Kuhl H, Sandow J, Busse R, Schini-Kerth VB (2001) Sex steroids used in hormonal treatment increase vascular procoagulant activity by inducing thrombin receptor (PAR-1) expression: role of the glucocorticoid receptor. Circulation 104(23): 28262831.

2. Köhler G, Faustmann TA, Gerlinger C, Seitz C, Mueck AO (2010) A dose ranging study to determine the efficacy and safety of 1, 2 and $4 \mathrm{mg}$ of dienogest daily for endometriosis. Int J Gynecol Obstet 108(1): 21-25.

3. Ruan X, Seeger H, Mueck A O (2003) The pharmacology of dienogest. Maturitas 71(4): 337344.

4. Teichmann A (2003) Pharmacology of estradiol valerate/dienogest. Climacteric 6(S2): 17-23.

5. Strowitzki T, Faustmann T, Gerlinger C, Seitz C (2010) Dienogest in the treatment of endometriosis associated pelvic pain: a 12 week randomised, double-blind, placebo-controlled study. Eur J Obstet Gynecol Reprod Biol 151(2): 193-198.

6. Sasagawa S, Shimizu Y, Kami H, Takeuchi T, Mita S, et al. (2008) Dienogest is a selective progesterone receptor agonist in transactivation analysis with potent oral endometrial activity due to its efficient pharmacokinetic profile. Steroids 73(2): 222-231.

7. Sasagawa S, Shimizu Y, Nagaoka T, Tokado H, Imada $\mathrm{K}$, et al. (2008) Dienogest, a selective progestin, reduces plasma estradiol level through induction of apoptosis of granulosa cells in the ovarian dominant follicle without follicle-stimulating hormone suppression in monkeys. J Endocrinol invest 31(7): 636-641. 
8. Katayama H, Katayama T, Uematsu K, Hiratsuka M, Kiyomura M, et al. (2010) Effect of dienogest administration on angiogenesis and hemodynamics in a rat endometrial auto graft model. Hum Reprod 25(11): 2851-2858.

9. Strowitzki T, Marr J, Gerlinger C, Faustmann T, Seitz C (2010) Dienogest is as effective as leuprolide acetate in treating the painful symptoms of endometriosis: a 24 week, randomized, multicentre, open-label trial. Hum Reprod 25(3): 633-641.

10. Fauconnier A, Dallongeville E, Huchon C, Ville Y, Falissard B (2009) Measurement of acute pelvic pain intensity in gynecology; a comparison of five methods. Obstet Gynecol 113(2): 260-269.

11. Schlaff WD, Carson SA, Luciano A, Ross D, Bergqvist A (2006) A subcutaneous injection of depot medroxyprogesterone acetate compared with leuprolide acetate in the treatment of endometriosis-associated pain. Fertil Steril 85(2): 314-325.

12. Barbieri RL (1992) Hormone treatment of endometriosis: the estrogen threshold hypothesis (review). Am J Obstet Gynecol 166(2): 740-745.

13. Pickersgill A (1998) GnRH agonists and add-back therapy: Is there a perfect combination? Br J Obstet Gynecol 105(5): 475-485.

14. Vercellini P, Bracco B, Mosconi $P$, Roberto A, Alberico D, et al. (2016) Norethindrone acetate or dienogest for the treatment of symptomatic endometriosis: a before and after study. Fertil Steril 105(3): 734-743.

15. Klipping C, Duijkers I, Faustmann T, Klein SF, Schuett B (2010) Pharmacodynamic study of four oral dosages of dienogest. Fertil Steril 94(4): S181.

16. Moore C, Carol W, Graser T, Mellinger U, Walter F (1999) Influence of dienogest on ovulation in young fertile women. Clin Drug Invest 18(4): 271-278.
17. Schindler AE (2011) Dienogest in long-term treatment of endometriosis. Int J of Women's Health 3: 175-184.

18. Momoeda M, Taketani Y, Terakawa N, Takesh ASO, Tsutani K (2007) Randomized double-blind, multicentre, parallel-group dose-response study of dienogest in patients with endometriosis. Jpn Pharmacol Ther 35(7): 769-783.

19. Petraglia F, Hornung D, Seitz C, Faustmann T, Gerlinger C, et al. (2012) Reduced pelvic pain in women with endometriosis: efficacy of long term dienogest treatment. Arch Gynecol Obstet 285(1): 167-173.

20. Momoeda M, Harada T, Terakawa N, Aso T, Fukunaga M, et al. (2009) Long term treatment of dienogest for the treatment of endometriosis. J Obstet Gynaecol Res 35(6): 1069-1076.

21. Whalen KL, Rose R (2011) Estradiol Valerate/Dienogest: a novel oral contraceptive. Ann Pharmacother 45(10): 1256-1261.

22. Fruzzeti F, Bitzer J (2010) Review of clinical experience with estradiol in combined oral contraceptives. Contraception 81(1): 8-15.

23. Rafie S, Borgelt L, Koepf ER, Temple-Cooper ME, Lehman KJ (2013) Novel oral contraceptive for heavy menstrual bleeding: estradiol valerate and dienogest. Int J Women's Health 5: 313-321.

24. American College of Obstetricians and Gynecologists (2001) ACOG practice bulletin: management of anovulatory bleeding. Int J Gynaecol Obstet 72(3): 263-271.

25. Von Schoultz B (2003) Clinical efficacy and safety of combined estradiol valerate and dienogest: a new no-bleed treatment. Climacteric 6(S2): 24-32. 